\title{
ELECTRICAL PROPERTIES AND POWER CONSIDERATIONS OF A PIEZOELECTRIC ACTUATOR
}

\author{
T. Jordan*, Z. Ounaies**, J. Tripp*, and P. Tcheng* \\ * NASA-Langley Research Center, Hampton, VA 23681, USA \\ ** ICASE, NASA-Langley Research Center, Hampton, VA 23681, USA
}

\begin{abstract}
This paper assesses the electrical characteristics of piezoelectric wafers for use in aeronautical applications such as active noise control in aircraft. Determination of capacitive behavior and power consumption is necessary to optimize the system configuration and to design efficient driving electronics. Empirical relations are developed from experimental data to predict the capacitance and loss tangent of a PZT5A ceramic as nonlinear functions of both applied peak voltage and driving frequency. Power consumed by the PZT is the rate of energy required to excite the piezoelectric system along with power dissipated due to dielectric loss and mechanical and structural damping. Overall power consumption is thus quantified as a function of peak applied voltage and driving frequency. It was demonstrated that by incorporating the variation of capacitance and power loss with voltage and frequency, satisfactory estimates of power requirements can be obtained. These relations allow general guidelines in selection and application of piezoelectric actuators and driving electronics for active control applications.
\end{abstract}

\section{INTRODUCTION}

The potential of piezoelectric ceramics as actuators and sensors has been widely documented in applications ranging from aerospace to biomedical. One of the areas that incorporates the use of these materials at NASA LaRC is the area of active noise and vibration control [1-3]. In this application, the targeted fundamental frequency is between $50-100 \mathrm{~Hz}$, with the next higher modes ranging up to $400-600 \mathrm{~Hz}$. These are higher frequencies than in structural or aeroelasticity control because of the pressurized or pre-loaded aircraft cabin/fuselage. Practical limitations such as acceptable excitation voltages, mechanical durability, coupling to the control structure and control system complexity and stability are driving research for sensor and actuator improvement. A critical issue that arises when using surface mounted transducers is the piezoelectric power consumption necessary to drive them. Applications are found to depend upon the electrical characteristics of the PZT transducers, namely the capacitive and resistive behavior of the actuator, which in turn affect their power consumption characteristics.

A number of researchers have investigated the power consumption characteristics of PZT actuators used to excite a host structure [4-6] and found a coupling between the mechanical motion of the structure and the electrical characteristics of the piezoelectric actuator. Research by Brennan and McGowan [7] shows that the power consumption of piezoelectric materials used for active vibration control is independent of the mechanical motion of the host structure when the structure is completely controlled. From these findings, they conclude that the power requirements of the piezoelectric actuator are only dependent upon its geometry and material properties, and the driving voltage and frequency of the control signal. They also note a linear variation of the capacitance of the actuator with the applied field. This is a phenomenon that is rarely discussed in the literature, but it is important to identify since assuming a constant capacitance and loss tangent can lead to large errors in predicting power requirements for driving 
the piezoelectric wafers. Their definition of effective capacitance includes both a dielectric and piezoelectric term, which is a departure from a traditional definition of capacitance.

In the present work, we extend the voltage and frequency ranges used in [7] and investigate both the capacitance and the loss tangent of the PZT as a function of driving voltage and frequency. We present a method for predicting power consumption given a wider range of applied voltage and frequency, and we identify two regimes for the capacitive and resistive behavior of the piezoelectric actuators. We also show that the behavior may not be simply a linear relationship, but rather can be more complex depending on the frequency regime in which the actuator is operating.

\section{EXPERIMENTAL}

To measure the power consumed by a PZT wafer (PZT 5A, 1.5" x 0.5" x 0.008"), a sinusoidal driving voltage $\mathrm{v}(\mathrm{t})$ was applied to the wafer. The peak amplitude and frequency of the voltage were controlled from a function generator. The peak voltage was varied from $20-$ 200 volts and the frequency from $10-400 \mathrm{~Hz}$. The PZT wafer was modeled as a resistor $\mathrm{R}_{\mathrm{c}}$ and capacitor $C$ in series (figure 1 ). A $1 \mathrm{k} \Omega$ resistor $R_{s}$ was placed in series with the wafer and the voltage across this resistor was monitored to determine the current $i(t)$ through the circuit. Both the driving voltage $\mathrm{v}(\mathrm{t})$ and the voltage across the resistor were routed to a digitizing oscilloscope where they could be visually monitored and downloaded to a PC for further investigation. The amplitude and phase of the signals were determined and the power consumed by the PZT was calculated. At higher voltages, the effect of the PZT's non-linear capacitance was evident by the distortion of the circuit loop current.

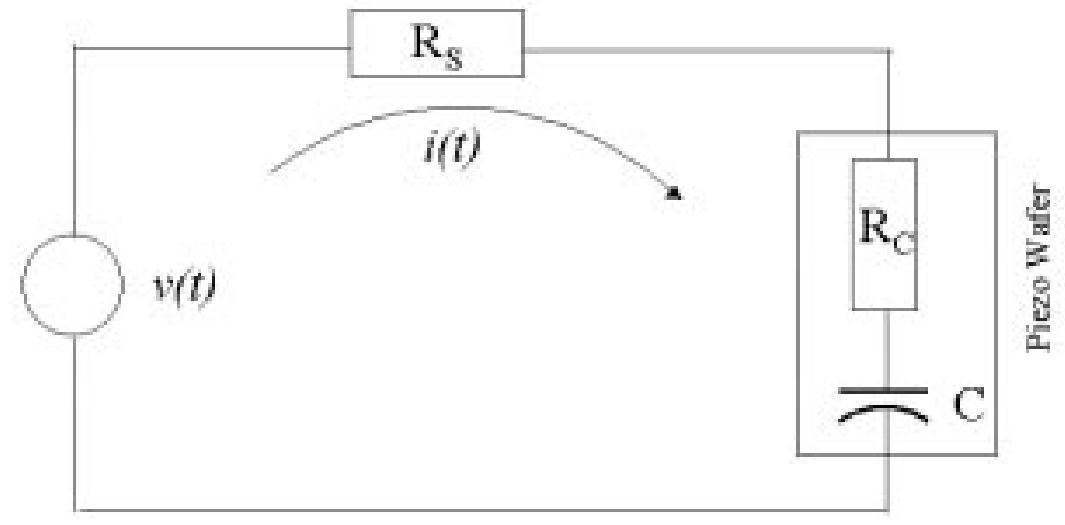

Figure 1. Piezoelectric wafer test circuit

Once digitized, the data was fit to the following third-order polynomial model:

$$
v(t)=v_{0}+c_{1} t+\left(R_{c}+R_{s}\right) i(t)+a_{1} q+a_{2} q^{2}+a_{3} q^{3}+a_{4} i q^{2}
$$

The $\mathrm{v}_{0}$ and $\mathrm{c}_{1} \mathrm{t}$ terms denote measurement bias errors. The terms with $\mathrm{q}$ represent the voltage across $\mathrm{C}$, with the higher order terms in q required due to the non-linear electrical characteristics of the PZT wafer. A more extensive discussion of this analysis is to be published in a forthcoming paper.

The resistive component of the piezoelectric wafer $\mathrm{R}_{\mathrm{c}}$ is derived using a least-square estimate from equation 1 . An equivalent capacitance $\mathrm{C}_{\mathrm{eq}}$ is defined as the ratio of the charge $\mathrm{q}$ and the RMS value of the voltage across the capacitance, and from equation (1) the charge can be solved as a function of the voltage across $\mathrm{C}$. 
Based on figure 1, it can be shown that the loss tangent $\tan \delta$ is given by:

$$
\tan \delta=2 \pi f \mathrm{C}_{\mathrm{eq}} \mathrm{R}_{\mathrm{c}}
$$

\section{RESULTS}

Figure 2 shows the measured capacitance as a function of peak driving voltage for various frequencies. A third order polynomial was used to fit the data, resulting in an expression of capacitance given by:

$$
\mathrm{C}=\mathrm{C}_{0}+\mathrm{C}_{1} \mathrm{~V}+\mathrm{C}_{2} \mathrm{~V}^{2}+\mathrm{C}_{3} \mathrm{~V}^{3}
$$

Where the $C_{n}$ 's are a function of frequency and are given in table 1 .

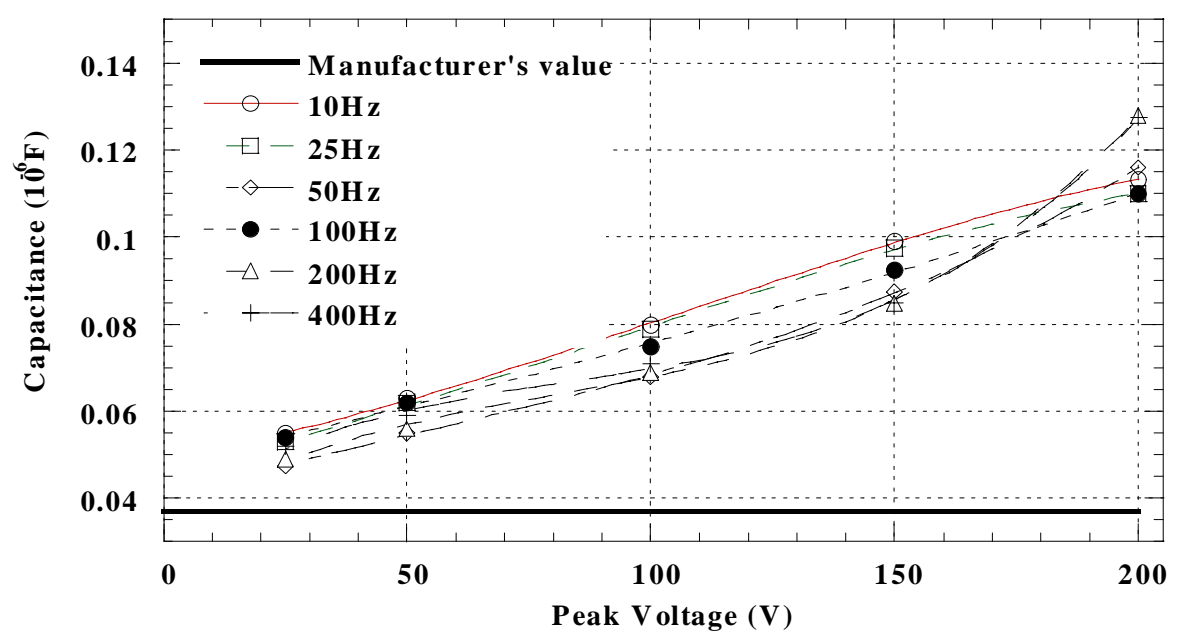

Figure 2. Measured and estimated capacitance as function of peak voltage.

Table 1. Third-order polynomial coefficients (equation3).

\begin{tabular}{c|cccc}
\hline Frequency $(\mathrm{Hz})$ & $C_{0}$ & $C_{1}$ & $C_{2}$ & $C_{3}$ \\
\hline 10 & 0.04951 & 0.000185 & $1.798 \mathrm{e}-6$ & $-5.654 \mathrm{e}-9$ \\
25 & 0.04646 & 0.000240 & $1.437 \mathrm{e}-6$ & $-5.235 \mathrm{e}-9$ \\
50 & 0.04012 & 0.000328 & $-1.152 \mathrm{e}-6$ & $7.049 \mathrm{e}-9$ \\
100 & 0.04783 & 0.000257 & $1.882 \mathrm{e}-7$ & $4.262 \mathrm{e}-10$ \\
200 & 0.03500 & 0.000671 & $-5.787 \mathrm{e}-6$ & $2.378 \mathrm{e}-8$ \\
400 & 0.03696 & 0.000729 & $-6.629 \mathrm{e}-6$ & $2.620 \mathrm{e}-8$ \\
\hline
\end{tabular}

Also shown in figure 2 is the capacitance value of the wafer as measured with a conventional LCR meter at $1 \mathrm{kHz}$ and low excitation voltage. This value of capacitance is normally held to be valid at varying frequencies and voltages. The measurement of the capacitance clearly shows an increase of the value as the driving voltage is stepped up. This is a phenomenon that is rarely mentioned $[7,8]$ and is little understood. It can however result in large errors in estimating power requirements if not taken into account.

In figure 3 , the capacitance $\mathrm{C}$ is shown as a function of frequency for each peak voltage. It is noted that below a certain frequency, $\mathrm{C}$ varies linearly with frequency, whereas above it, it 
varies non-linearly with frequency. At any given voltage, the value of the capacitance $\mathrm{C}$ changes more significantly below $50 \mathrm{~Hz}$ than it does between 50 and $400 \mathrm{~Hz}$.

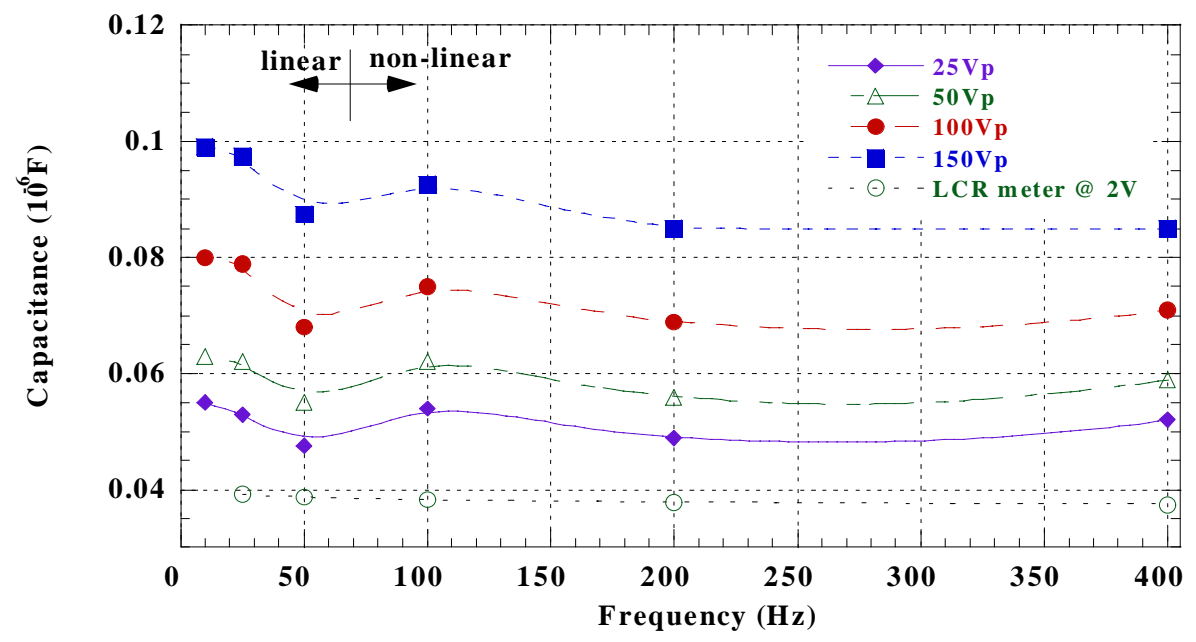

Figure 3. Measured and estimated capacitance as a function of frequency

Figure 4 shows the measured resistance $\mathrm{R}$, as a function of peak driving voltage for various frequencies. $\mathrm{R}$ is seen to vary with the driving voltage. A third order polynomial was used to fit the data, resulting in an expression of resistance given by:

$$
\mathrm{R}=\mathrm{R}_{0}+\mathrm{R}_{1} \mathrm{~V}+\mathrm{R}_{2} \mathrm{~V}^{2}+\mathrm{R}_{3} \mathrm{~V}^{3}
$$

Where the $\mathrm{R}_{\mathrm{n}}$ 's are a function of frequency and are given in table 2 .

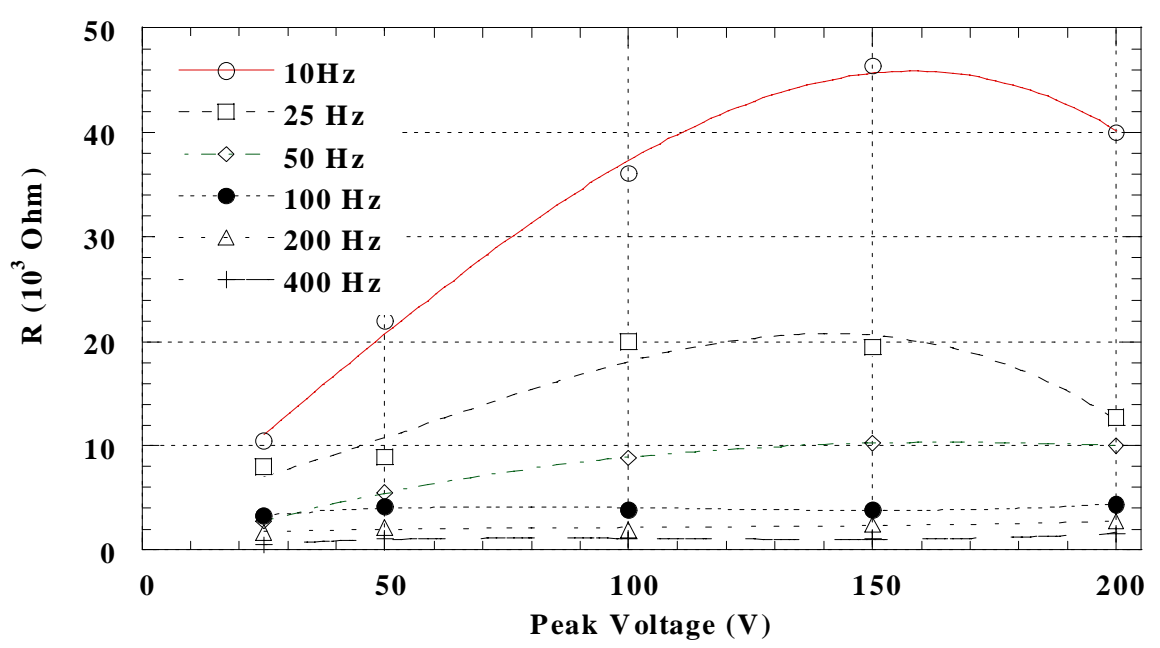

Figure 4. Measured and estimated resistance of a piezoelectric wafer. 
As expected, as the frequency increases, the resistance of the PZT wafer decreases (conductivity effects become more apparent).

Table 2. Third-order polynomial coefficients (equation 4).

\begin{tabular}{c|cccc}
\hline Frequency $(\mathrm{Hz})$ & $R_{0}$ & $R_{1}$ & $R_{2}$ & $R_{3}$ \\
\hline 10 & 1.40080 & 0.376690 & 0.00056529 & $-7.3972 \mathrm{e}-6$ \\
25 & 4.40730 & 0.080553 & 0.00132790 & $-7.6407 \mathrm{e}-6$ \\
50 & -0.59757 & 0.152651 & -0.00064813 & $7.5099 \mathrm{e}-7$ \\
100 & 2.25230 & 0.060235 & -0.00059746 & $1.7587 \mathrm{e}-6$ \\
200 & 1.45560 & 0.015823 & 0.0001260 & $4.0397 \mathrm{e}-7$ \\
400 & -0.27937 & 0.044703 & -0.00042829 & $1.2683 \mathrm{e}-6$ \\
\hline
\end{tabular}

The loss tangent of the wafer is calculated from the resistance data using equation 2 . Figure 5 shows the behavior of $\tan \delta$ with both voltage and frequency. The loss tangent increases as much as three times at any given frequency in the voltage range considered.

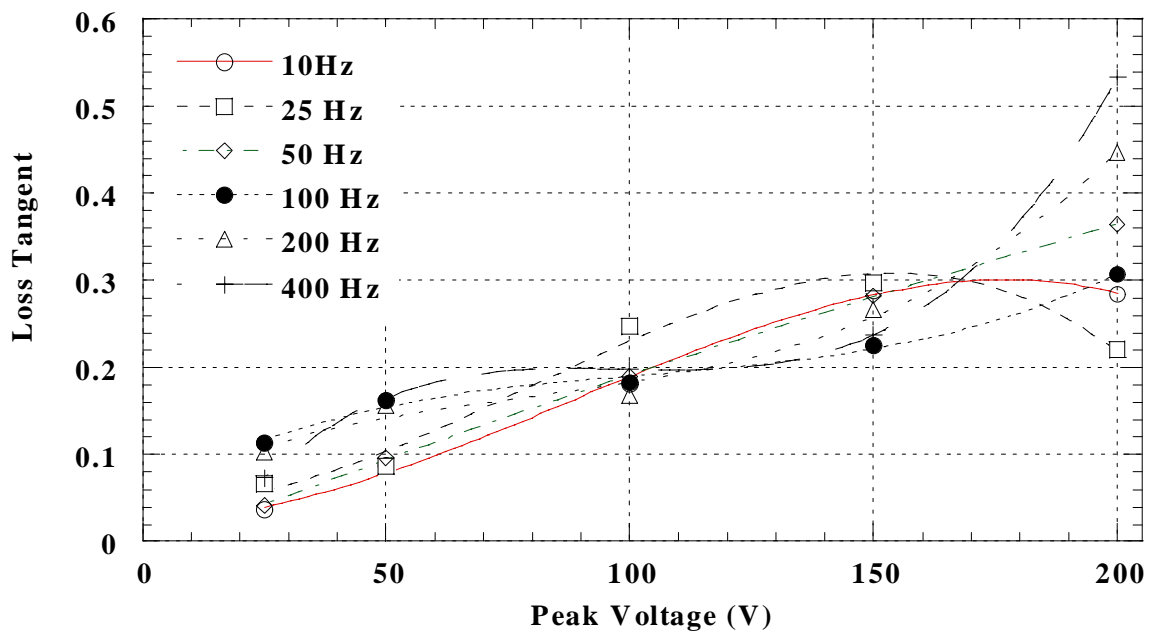

Figure 5. Calculated (equation 2) and estimated $\tan \delta$.

Using the experimental data, the mean power consumed by the piezoelectric wafer was calculated as

$$
\mathrm{P}=\mathrm{I}_{\mathrm{rms}} \mathrm{V}_{\mathrm{rms}} \cos \theta
$$

Where $V_{\mathrm{rms}}=\mathrm{Vp} / \sqrt{2}, \mathrm{I}_{\mathrm{rms}}=\mathrm{Ip} / \sqrt{2}$, and $\theta$ is the phase shift between the voltage and the current. An estimate of the power consumed can be derived based on the RC series circuit used to model the piezoelectric wafer (figure 1) such that:

$$
\mathrm{P}=2 \pi \mathrm{fC} \tan \delta \mathrm{V}_{\mathrm{rms}}^{2}
$$

Both calculated power (from measured values) and estimated power (based on equation 6) are shown in figure 6 below, as a function of peak voltage and frequency. 


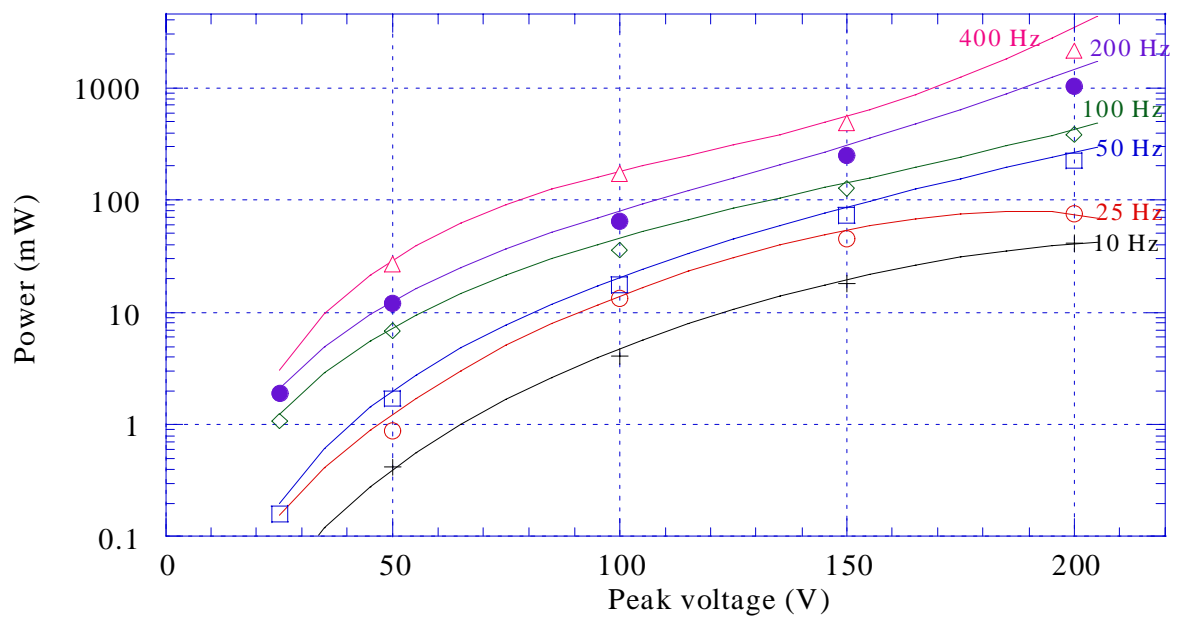

Figure 6. Estimated and measured power consumption of the piezoelectric wafer

Figure 6 shows good agreement with the measured values of power, especially below $150 \mathrm{Vp}$. It is surmised that at voltages above $150 \mathrm{Vp}$, the PZT wafer is operating outside the linear range of the piezoelectric response, where switching of the dipoles commences.

\section{SUMMARY}

The capacitance and loss tangent of a piezoelectric wafer were characterized as a function of driving field and frequency. An analytical model based on RC series circuit was used to estimate the capacitance and resistance of the piezoelectric wafer. Mathematical relations were then developed to relate the capacitance and resistance values to the peak driving voltage and frequency. These values were used to predict the power consumed by the wafer and gave very good estimates when compared to measured values.

In future studies, the electromechanical efficiency of the piezoelectric actuators will be investigated in this frequency range.

\section{REFERENCES}

1. Lyle, K. H. and Silcox, R. J., S. A. E. Transactions 104, p.180 (1996).

2. Fuller, C. R. and Silcox, R. J., J. of the Acoustical Society of America 91, p.519 (1992).

3. Fuller, C. and von Flotow, A., J. of Sound and Vibrations 203, p. 745 (1997).

4. Stein, S. C. and Rogers, C. A., J. of the Acoustical Society of America 96, p.1598 (1994).

5. Liang, C., Sun, F. P., and Rogers, C. A., J. of Intelligent Mater. Syst. And Struct. 5, p.12 (1994).

6. Hagood, N. W., Chung, W. H., and von Flotow, A., J. of Intelligent Mater. Syst. And Struct. 3, p.327 (1990).

7. Brennan, M. C. and McGowan, A-M., Proceedings of SPIE: Smart Structures and Materials 3039, p.660 (1997).

8. Warkentin, D. J. and Crawley, E. F., MIT Space Engineering Research Center 4-95, (1995). 